\title{
The Review of using Unified BPM Cycle for Public Credit Recovery Activites
}

By Nipuni Sashanka Perera Abstract- Business Process Management (BPM) is a combination of Information Technology and management science, which applies to improve business process in order to improve operational excellence and business performances [1] leading to process automation. This review article based is on a case study in application of business governance in public sector organization, which was conducted by Abimael R. Do Nascimento, Roquemar de Lima Baldam, Lourenço Costa and Thalmo de Paiva Coelho Junior in 2018. The article analyzes the implementation of unified BPM in operational activities in a federal public advocacy body with evaluating corporate governance practices of the process. The study used mix method approach to gather data and to analyze them. Findings revealed the requirement of corporate governance practices, prioritizing BMP and auditing process.

Keywords: business process management, bpm cycle, governance, operational excellence, public sector.

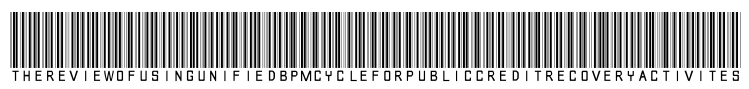

Strictly as per the compliance and regulations of:

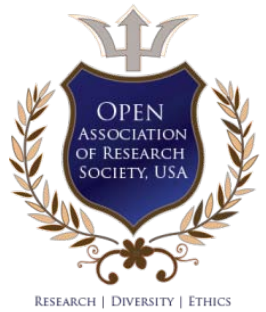

(C) 2020. Nipuni Sashanka Perera. This is a research/review paper, distributed under the terms of the Creative Commons Attribution-Noncommercial 3.0 Unported License http://creativecommons.org/licenses/by-nc/3.0/), permitting all non-commercial use, distribution, and reproduction in any medium, provided the original work is properly cited. 


\title{
The Review of using Unified BPM Cycle for Public Credit Recovery Activites
}

\author{
Nipuni Sashanka Perera
}

\begin{abstract}
Business Process Management (BPM) is a combination of Information Technology and management science, which applies to improve business process in order to improve operational excellence and business performances [1] leading to process automation. This review article based is on a case study in application of business governance in public sector organization, which was conducted by Abimael R. Do Nascimento, Roquemar de Lima Baldam, Lourenço Costa and Thalmo de Paiva Coelho Junior in 2018. The article analyzes the implementation of unified BPM in operational activities in a federal public advocacy body with evaluating corporate governance practices of the process. The study used mix method approach to gather data and to analyze them. Findings revealed the requirement of corporate governance practices, prioritizing BMP and auditing process.
\end{abstract}

Keywords: business process management, bpm cycle, governance, operational excellence, public sector.

\section{INTRODUCTION}

B usiness Process management (BPM) is a compound of data restructuring and overseeing which applies to achieve or produce something better than its earlier production in business process to upgrade business operations and execution with the help of information technology [1]. Business process management is development of workflow management, which lead to automate business process. Business Process Management (BPM) has got impressive consideration. Moreover, because of its potential for fundamentally operational efficiency and cost saving are expanded. In addition, an ample of Business Process Management (BPM) systems, such as conventional programing systems. These systems are driven by express procedure plans used to authorize and supervise operational business process [1]. Business Process Management (BPM) has a more extensive degree in business operation. The aims of Business Process Management (BPM) is improving operational business forms without using new technology. For an example, displaying and breaking down of a business procedure may be the best way to decrease costs while improving operational services. Business Process Management (BPM) regularly connect with the programs to supervise, control and support operational procedures. This is the main point of Work Flow Management (WFM). In any case, customary Work Flow

Author: Department of Research \& Graduate Studies Sri Lanka Institute of Information Technology, Colombo, Sri Lanka.

e-mail: nipunisashanka@gmail.com
Management (WFM) innovation focused on the mechanization of business forms fairly.

Business Process Management (BPM) examine a plenty of strategies, systems, and devices. Operational business forms are helped to structure, empower, manage and examine of above strategies, systems and devices. This article tries to discuss the outcomes and outlines of the survey, which was conducted by Abimael Rondon Do Nascimento, Roquemar de Lima Baldam, Lourenço Costa and Thalmo de Paiva Coelho Junior 2020 in "Applications of business governance and the Unified BPM Cycle in public credit recovery activities".

Furthermore, to improve the performance of public sector and adoption of innovative practices are the most important points of this article. Simply adopting innovative practices means transferring principles, techniques, which are adopt in private sector to the public sector. It increased the demand for better public services and it provides less expensive services to the public. When considering about the government systems impairment in the operations, this approach provides solutions such as effectiveness, efficiency and logical structures for the people to behave in rational ways to government systems impaired operations [2].

As the theoretical reference, authors used governance and operational activities. Simply, the system, metrics and roles and responsibilities can use to measure the improving of implementation. Furthermore, it can be identified as method that used to govern processes of the organization. The main responsibility of governance is to establish management practices. Similarly, it helps to increase probability of success. Furthermore, to maintain alignment of organizational objectives, execution of those objectives, manage them and through that improvement of the dayto- day activities [2].

To conduct the research authors chose the Federal Attorney in the State of Espirito Santo. They determined liquidity and credit certainty of public authorities as well as foundations [2].

\section{Literature And Theoritical Review}

Significantly, less has been written about the public sector broad writing in business process management, and what has been composed has been general. Subsequently, there is a disarray among public authorities on how business process the board ideas 
ought to be executed [3]. Public organizations should change their operational structures fundamentally just as their undertaking frameworks to actualize business process the board ideas effectively [3]. Public sector supervision and administration in these days tries to build the adequacy of the inward regulatory procedures offer higher level in administration towards residents or citizens. Business Process Management comprises of distinguishing and upgrading business forms. Furthermore, to increase business forms powerfully, effectively and progressively equipped for adjusting an ever-changing public sector associations. In public sector, specialists have colossal assistance covers on the grounds that the capacity to share information about business forms is restricted bringing about a plenty of waste, costs, when performing activities [4].

a) BPM in public sector

BPM is a methodology that devoted process driven organizations. It is focused on analyzing, improving and controlling procedures. Similarly, coordinating individuals, procedures and innovations are targeted. In that BPM is regularly applied and confirmed in the private sector. Though, it uses in private sector, it is not applied in public sector commonly. Public organizations need to upgrade adequacy and raise consumer loyalty. So most of the organizations implement strategies and methods which utilized the administration of profit oriented organizations. Effective process management decreases cost. Furthermore, residence is given high quality of services. Similarly, the productive utilization of assets is increased. Assets are included data and communication systems supporting government activities. [2].

Since mid-90, the public sector has been confronted difficulties and desires. Expanding customer requirements, IT improvements, competition among economies are required effective operation in public sector with innovations in a cost effective way [5]. This reality may also affirm the solid enthusiasm for the system of BPM execution. Moreover, enormous number of papers discussed about this exploration issue, and recommends the need in building up a BPM system for the public sector organizations [6]. The study focused local government capabilities of business process management and the findings revealed that this method is under developing stage in public sector. Similarly, at the medium stage there are governance, information technology, strategic alignment, culture and people [6]. Dysfunction should be the investigation of public setting. Furthermore, it is important to consider multifaceted nature and the size of the public sector exercises that make it hard to gather and procedure data about administrations, execution and make it trying to investigate public segment's wellsprings of information and improve the inner procedures [7]. Majority of literatures suggests that majority of public sector associations are at initial level of business process management life cycle. In this level, organizations are considered process design, which consist with modules used to gain knowledge of operational process and capabilities [7]. When moving to innovative practices adoption size of the public sector and complexity of activities are important areas to be concerned. Similarly, those factors are difficult to collect, and process. Furthermore, knowledge source of public sector is difficult to identify and it makes internal process improvement difficult [2].

Organizational efficiency and effectiveness related problems increased because of the uncontrolled processes they used. The reason is those activities are repetitive and identified as operational activities. Moreover, to meet financial, political and social challengers' public organizations should think differently, and adapt and change their processes. Through implementing BPM methods organizations are focusing on improvement of their day-to-day performance. BPM

promotes knowledge, management, process improvement and process alignment with objectives of the organization. Therefore, BPM is the possible answer to the above challengers [2].

Similarly, in the perspective of public administration, implementing BPM methods will have greater possibility to increase effectiveness and efficiency. So public sector pays their attention on implementing BPM processes in the organizations. When implementing PBM methods organizations have to consider about several factors such as complexity of operational activities and diversity of not only public organizations but also private organizations. Those factors should consider about both internally and externally [2].

The particular study focused business governance and business process management in public sector, many of literatures argued that there is a contextual gap in the field of the study, which is business process and governance principles in public sector [8]. Contends that most issues with hierarchical productivity and viability have fundamental uncontrolled procedures, and specific gathering of exercises that are tedious and allude as operational exercises. Public sector should re-examine, adjust and change procedures to overcome new and developing financial, social and political threats and challenges [8].

To achieve their objective, they performed a theoretical survey of BPM. Unified BPM Cycle model chosen and implemented. Through the findings and the analysis of results, authors identified construction structure to corporate governance with the implementation and maintenance activities. Authors prioritized main processes, analyzing and modelling of processes, how those processes implement in an 
effective method, how to optimize processes and finally how to monitor and audit those processes [2].

\section{b) Governnce and operations}

Before implementing a BPM, it is important to get better understanding about organization. It includes both external and internal environment, main processes within organization, impairments in the operations of a system and its most relevant characteristics. Then, corporate governance should be established as initial measures for the successful planning of Business Process Management actions [2].

Furthermore, the experience level of the team, state the project implementation management process, organize and provide both implementation and maintenance actions to Business Process Management. In the perspective of scare resources, prioritize the processes, modelling the processes and create plans for optimization of processes, specify their sources for its success [2].

Moreover, get appropriate comprehension of the setting in which the organized procedures are embedded, recognizable proof of potential holes in comprehension and execution, information on the present condition of these procedures, distinguishing proof of opportunities for development and use of the outlining strategy picked to formalize. At last, implemented processes, monitor the execution, checked and confirmed the previously planned audit procedure with the current procedure is must [2].

The paper discussed governance practices in public operation. Setup governance practices are key successful factor in business process management [9]. The term governance describes solutions for agency problems and it origins with separation of ownership of the business and control. The paper analyses the application of governance concept with business process management in order to gain maximum benefits of operation with transparency [9]. Best practices in business context is important for achievement of business forms. The term business process administration alludes to the course, coordination, and control of people, gatherings, or associations that are at any rate self-governing: that is, not legitimately dependent upon the equivalent various levelled authority [10].

\section{c) From work flow to unified BPM}

The mid 1980s saw development in work process items for human-confronting forms, for example, the endorsement stream for a huge buy request. Incorporation between big business applications and these work process forms must be exceptionally constructed, so they were normally costly and unyielding. Moreover, after it has all said and done, these work process items had an exceptionally huge effect. In his book, The World is Flat; Thomas Friedman distinguished work process as the number three driver for the new level world by empowering an "unrefined establishment of a totally different worldwide stage for coordinated effort." During a similar period, venture application joining (EAl) items developed to improve framework to-framework interchanges so the information in one framework could consequently show up in another without the requirement for a human to re-type it. In spite of the fact that there was some crossfertilization of highlights and capacities between the two, the two arrangements of items kept on developing freely. Indeed, even as of late as 2007, driving industry investigators were dividing the business procedure the executives advertise into human-driven, framework driven, and report driven. Unadulterated play BPM sellers - the individuals who had developed from the work process legacy-tended to the human-driven space, middleware and combination merchants tended to the framework driven market, and substance the board merchants tended to the record driven market [11].

This fragmentation was problematic for clients who found that as procedure activities develop they seldom fit inside the limits of one of the storehouses. Along these lines, largely, the underlying choice was an inhibitor in a more extensive rollout of the venture. Likewise, this implied most clients had three or four items with partners vested in each, acquiring superfluous individuals and political contemplations into dynamic. The outcome was that BPM was fundamentally constrained to departmental organizations. A BEA highlights development overview finished by about 1,200 business and IT experts all through 2007 indicated that only 18 percent of organizations were right now conveying venture wide BPM. Luckily, for clients, this circumstance quickly changed. In 2008, Oracle obtained BEA, and later IBM procured Lombardi. There was other solidification right now. 

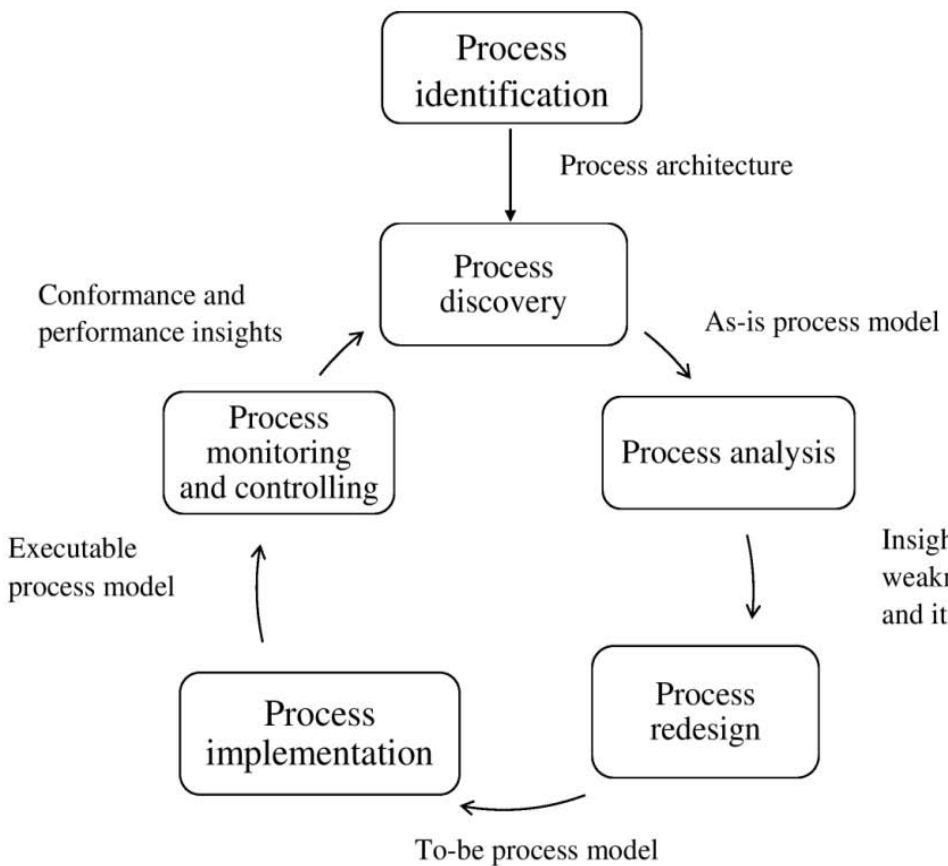

Insights on weaknesses and its impact

To-be process model

Source: (M. Dumas, M. La Rosa, J. Mendling, and H. Reijers, Fundamentals of Business Process Management, Heidelberg: Springer, 2016)

Fig. 1: Traditional BPM cycle

This combination moved the conversation of BPM's business request overlaid on a powerful venture grade SOA stage from the domain of a hypothetical design to genuine item contributions. This offered clients three essential advantages:

- The capacity to coordinate their procedures with any application or framework it expected to incorporate with, utilizing benchmarks based powerful and make sure about joining innovations

- The confirmation that the procedure would scale to any exhibition and volume necessities and that the innovation could be utilized for strategic procedures

- Enterprise-grade the board, organization, and checking apparatuses

Preceding this unification, BPM and business rules motor were distinctive item classes. BPM has an inalienable requirement for business rules and most unadulterated play BPM sellers tended to this with scripting, for the most part putting a businessaccommodating facade on top. Another advantage of this unification was the meeting up of BPM and business rules - with the main merchants giving combination at run time and a totally bound together experience traversing demonstrating, execution, and the board [11].

When consider about unified BPM model main focuse of Business Process Management is improving organizational performances through managing business processes. It indicates continuous improvement of processes in the organization and adding value to them. Through that organizations can make their processes efficient. Similarly, knowledge is sharing among the people in the organization. From the operations perspective BPM trying to identify the process, analyzing, designing, execution, monitoring and improvement of business process with the colllaboration of every resource in the organization. Simply every resource means people, applications and information sources. Furthermore, it consider about organizational implementation, performance control in the organization and leadership without limited their focus in analysis and modelling of the operations [11].

Unified BPM model provides numerous benefits to the organizations. It improves productivity and the service quality of the organization. It controls the cost of the organization. It improves both process and the compliance of the organization. It provides clear definition about roles and responsibilities of the employees within the organization and it will help to improve the employees' contribution to the organization. Furthermore, it will provide greater visibility of personal results of the employees. Organizations must understand how they are going to approach their methodology and how they are going to create a model, which is capable of guiding the organization actions to promote better understanding about Business Process Management. The results of this study provides a referenced Unified BPM Cycle model and it depicts the BPM lifecycle optimization. It consists with main four stages such as planning the process, analysis, modeling and optimization the process, implementation the process and monitoring the process [11]. 


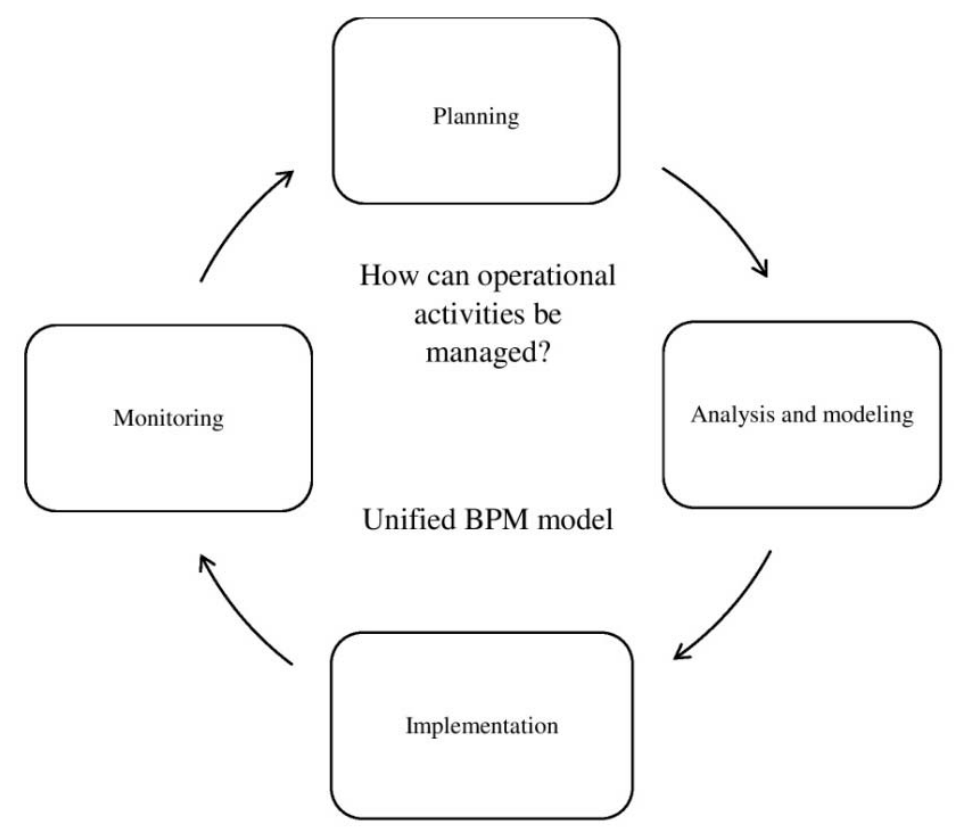

Source: (Abimael Rondon Do Nascimento, Roquemar de Lima Baldam, Lourenço Costa and Thalmo de Paiva Coelho Junior, "Applications of business governance and the Unified BPM Cycle in public credit recovery activities," Business Process Management Journal, pp. 312-330, 2020)

\section{Fig. 2: Unified BPM cycle}

Business Process Management is not an isolated discipline. It uses different techniques, tools and technologies when necessary. As an example lean thinking, BPR, procedure standardization, project and quality management and simulation of processes can be identified. Furthermore, multi-method approach needs to use for the application, which has several techniques and tools. It combines two distinct methods, including qualitative and quantitative methods. This study carried round activities and because of that action research, can be taken as most appropriate method as mentioned by the authors. That method has relation to solve collective problems because of that authors and research participants got the ability to engage in cooperative way. What's more, it encourage more adaptable origination and utilization of methods for examination and gave, through the activation that happened in the gathering forms, the catch of data in a more profound and increasingly sensible level [12].

The study conducted through "multi-method" approach, which is a combination of many of research tools. Multi method is mix method, which is consists of qualitative and quantitative data, methodologies, methods, and paradigms in a research study or set of related studies. The research method overcome various disadvantages in varicose research approaches [13]. The most proper research technique to help this methodology was activity inquire about, on account of its connection to tackling aggregate issues in which analysts and participants take part in an agreeable manner. Likewise, it encouraged the more adaptable origination and utilization of the methods for examination and gave, through the assembly that happened in the gathering forms, analysis information in deeper and realistic way.

At the initial step, the study focused on understanding organizational context, which consists with external and internal environments, stakeholders' expectations, internal controls mechanisms, process, critical characteristics and establishment of corporate governance principle to review and measure business process management activities and plans. The research method is clearly elaborated in Figure (Fig.3). 


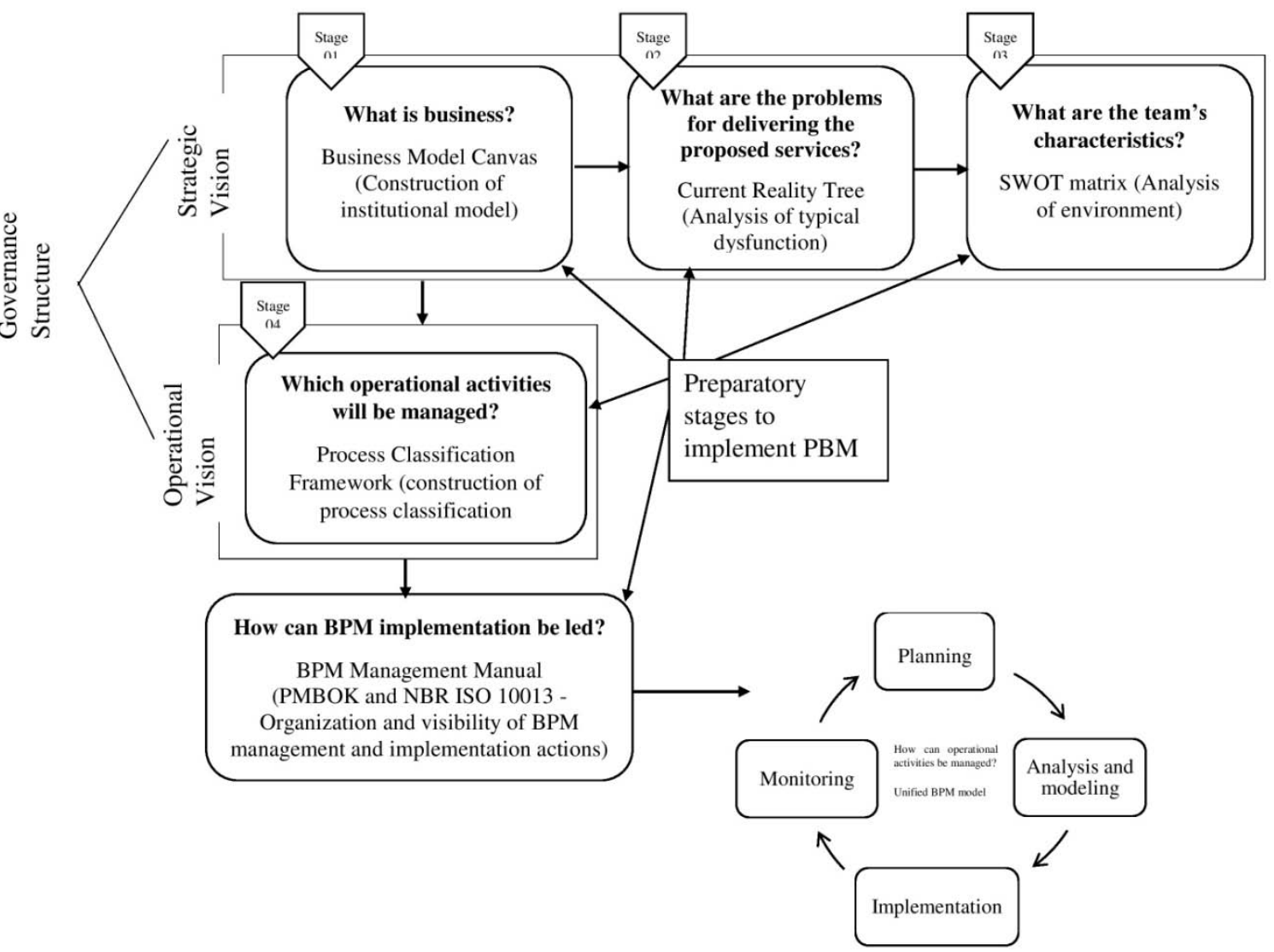

Source: (Abimael Rondon Do Nascimento, Roquemar de Lima Baldam, Lourenço Costa and Thalmo de Paiva Coelho Junior, "Applications of business governance and the Unified BPM Cycle in public credit recovery activities," Business Process Management Journal, pp. 312-330, 2020)

\section{Fig. 3: Structure of corporate governance}

Next, the study focused to investigate the level of engagement of teams in management and implementation of projects, organization of business process, optimizing process and allocating resources based on scarcity and prioritization of the work. Later the study aimed to identify performances gaps, current level of knowledge in process, identification of possibilities for improvement. Application of the diagramming method is chosen to formalize them.

Further study was based on Federal Attorney in the State of Espírito Santo. It represents the Federal Union judicially and extra judicially. The study concentrated on the normally authoritative operational exercises of credit collection and recovery. Moreover, an enormous portion of exercises performed by the body which is legitimate in nature and not repetitive.

\section{iil. Research Findings}

Authors collected data through preliminary consultations. They are expert in present practices of laws, ordinances, instructions and dispatches of administrative-managerial content relating to BPM. After comprehensive understanding of credit recovery business, the study has been come up with results of identification with 22 typical types of dysfunctions, which are lead to generate inefficacies (Refer Annexure 01). Further, the study revealed that there were eight positive internal strengths, two external opportunities or positive factors, seven internal weaknesses and eight external threats according to SWOT analysis, which was conducted by the author.

The analysis of BPM maturity was revealed that the particular public organization is at initial step of process management initiatives. Based on six factor survey which includes IT, Methods, Governance, People, Culture and Strategic alignment, BPM consumes considerable resources and the organization is at the initial stage of BPM maturity in surveyed six factors. Prioritization of BPM is at higher level in the particular public sector organization. Organizations face greater challenge of prioritizing business process because of limited resources and political decisions [14].

The study revealed that the requirement of documentation of the process and improvements are lead to curse grate visibility. The process, which implemented to be defined and the detailing is required. The reports were made accessible in a spot normal to the group in a corporate system condition. The modifications were overseen utilizing spreadsheets 
created for this reason, in this way guaranteeing command over the progressions made.

The observations and results acquired with the review of the procedures executed, when contrasted and the numbers got through the observing and dysfunction uncovered with the corporate governance structure. Affirmed need to execute in this stage of BPM usage, particularly when checking is centered on expost examination. In addition to above findings, the study revealed that the transaction cost of activities engages in business process management is at higher level and the study revealed new parameter allocating resources with credit recovery activities or even reveal undetected inefficiencies in the monitoring phase.

\section{Conclusion}

A commitment of this article, both focused in hypothetical and functional areas, are introduced an administration structure which represent exercises and procedures appropriate for public organization, that coordinates different activities required, by the distinguishing proof of association's finished extent with conveyance, by conveyance dysfunctions, exercise structures and how they ought to be overseen. This article does not cover a total and appropriate structure of public sector organization and the study is to fill the contextual and theoretical gap of the field of study.

Governance structure of public sector organization is lead to ensure transparency, accountability of process implemented. It likewise allowed elaboration of a durable structure for the characterization and the board of the organized procedures and other authoritative procedures, therefore fortifying the benefits of gathering procedures to catch data with profundity and authenticity. The simple method to be used to prioritize business process through set of credit recovery process instead of process-by-process comparison. Business and industry factors are evaluated processes in terms of efficiency, effectiveness and relevance by the stakeholders. This evaluation, is related to prioritization of business process. It serves as a foundation for the management decision-making process and improvement and redesign [15]. The away from of the jobs and duties of the parties who are associated with the prioritizing business process, accomplished from the recognizable proof of the present situation and its streamlining to ideal situation, and plan of charts, process manuals, operational guidelines and as well as reciprocal archives gave regular comprehension among the group with respect to the execution of these exercises, along these lines diminishing the varieties and vulnerabilities in their execution.

Based on the study findings and discussion, the study provides insights to develop and improve business process in public sector. There may not be seen numerous studies in public sector organizations with regarding to business process management and the paper provides border view of implementing business process in public sector embedded with corporate governance practices which ensure process efficiency to meet increasing requirements of general public. Six factor surveyed which is consists with IT, Governance, Culture, People, methods, strategic alignments are not at maturity levels and process manager can focus such factors carefully before implementing or improving existing business process. Based on the findings capacity and implementation of particular factors are at initial stages with compared to privet sector organizations. At last, the paper provides fruitful insights in public sector to apply business process management with corporate governance practices.

Finally, according to the above findings; study of the development and the application of execution indicators are suggested as future research [2].

\section{ACKnOWLeDgment}

It is with a great pleasure I pen my gratitude to Dr. Nuwan Kuruwitaarachchi for his veteran consultant and mentoring. Thank you for your understanding, motivation, guidance and enthusiasm and it made me determine and dedicating to write this review article.

\section{References Références Referencias}

1. W. M. P. Van der Aalst, "Business Process Management: A Comprehensive Survey," ISRN Software Engineering, p. 37, 2013.

2. Abimael R. Do Nascimento, Roquemar de Lima Baldam, Lourenço Costa and Thalmo de Paiva Coelho Junior, "Applications of business governanve and the unified BPM cycle in public credit recovery service," Business Process Management Journal, vol. 26, no. 1, pp. 312-331, 2020.

3. T. R. Gulledge and R. A. Sommer, "Business process management: public sector implications," Business Process Management Journal, vol. 08, no. 04, pp. 364-376. , 2002.

4. G. A. Papadopoulos, E. Kechagias, P. Legga and I. Tatsiopoulos, "Integrating Business Process Management with Public Sector," in Proceedings of the International Conference on Industrial Engineering and Operations Management, Paris, France, 2018.

5. J. Becker, L. Algermissen and B. Niehaves, "A procedure model for process oriented: EGovernment projects," Business Process Management, vol. 12, no. 1, p. 25, 2006.

6. B. Niehaves, R. Plattfaut and J. Becker, "Business process management capabilities in local 
governments: A multi-method study," Government Information Quarterly 30, pp. 217-225, 2013.

7. R. Gabryelczyk and A. Jurczuk, "Business process management in the public sector: explored and future research fields," in 9th Annual Conference of the EuroMed Academy of Business: Innovation, Entrepreneurship and Digital Ecosystems, Poland, 2016.

8. M. Rohloff, "Advances in business process management implementation based on a maturity assessment and best practice exchange," Information Systems and e-Business Management, vol. 09, no. 01, pp. 383-403, 2010.

9. J. Jeston and J. Nelis, Business Process Management: Practical Guidelines to Successful Implementations, Elsevier, 2006.

10. M. L. Markus, "Business Process Governance," Handbook on Business Process Management, pp. 201-222, 2010.

11. M. Das, "Enterprise System Journal," 1105 media inc., $12 \quad 10$ 2012. [Online]. Available:
https://esj.com/Articles/2012/12/10/Evolution-of-Uni fied-BPM.aspx?Page =2. [Accessed 24 March 2020].

12. M. Szelągowski, "Evolution of the BPM Lifecycle," in Communication Papers of the Federated Conference on Computer Science and Information Systems, 2018.

13. J. Byrne, "An Introduction to Mixed Method Research," Atlantic Research Centre for family work issues, p. 4, 2007.

14. J. Ohlsson, S. Han, F. Carpenhall and P. Johannesson, "Prioritizing Business Processes Improvement Initiatives: The Seco Tools Case," The Seco Tools Case, pp. 10.1007/978-3-319-078816 18, 2014.

15. J. Ohlsson, Shengnan and H. Bouwman, "The prioritization and categorization method (PCM) process evaluation at Ericsson: a case study," Business Process Management Journal, vol. 23, no. 02, pp.377-398, 2017.

\section{Anexture 01}

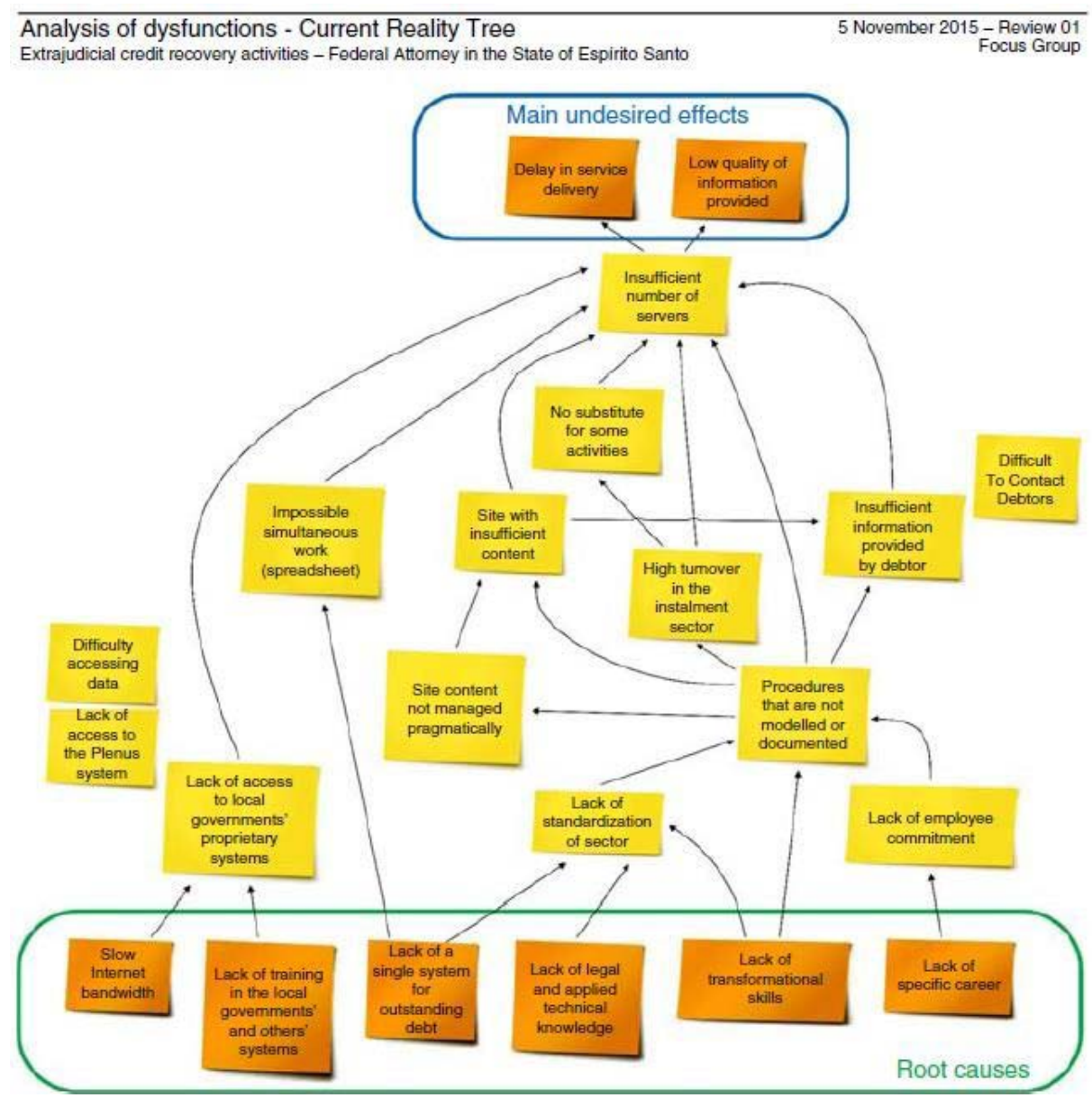

Source: (Abimael Rondon Do Nascimento, Roquemar de Lima Baldam, Lourenço Costa and Thalmo de Paiva Coelho Junior, "Applications of business governance and the Unified BPM Cycle in public credit recovery activities," Business Process Management Journal, pp. 312-330, 2020)

Fig. 4: Analysis of dysfunction- Current Reality Tree 